The Tension Mounts:

Will This Be The Year For The First Commercial $\mathrm{X}$-Ray Microcalorimeter?

Bob Compton, Microscopy Today microtoday.rdc@mindspring.com

With the upcoming Microscopy \& Microanalysis Conference only a few weeks away, the question that is in the mind of many industry experts is: "Will this be the meeting where the first commercial x-ray microcalorimeter is introduced?" If it is, you can bet your bottom dollar that there will be a major upheaval in the high resolution x-ray EDS business because of the ten- to fifty-fold improvement in $\mathrm{X}$-ray resolution promised by this extraordinary device.

\section{A little history}

Proposed by Lesyna et al. in 1993, and then initially demonstrated by Irwin et al. in 1996, the super-sensitive x-ray detector technology gained full speed in 1997 with the publication of the paper entitled "High-energy-resolution microcalorimeter spectrometer for x-ray microanalysis". Published by NIST experts Wollman, Irwin, Hilton, Dulcie, Newbury and Martinis, from both the Gaithersburg and Boulder facilities, this classic 25-page paper described the development, design and testing of a prototype unit.

The door is opened to commercialization

Then in July of 1999, NIST announced that co-exclusive licenses were granted to EDAX of Mahwah, NJ and NORAN Instruments of Middleton, $W I$ for the commercialization of the revolutionary microcalorimeter-based $\mathrm{X}$-ray detector. What the excitement is all about is that the new detector may have an energy resolution of 2 electron volts, some 50 time better than conventional semiconductor based detector. In fact this detector is claimed to have real time spectral acquisition similar to the popular EDS systems but with the resolution of a WDS spectrometers (see following figure).

This extraordinary resolution will enable chemical analysis of particles that are difficult, if not impossible, to study with current detectors. Of particular importance to the semiconductor industry, is that the detector will permit the chemical analysis of extremely small particles that contaminate silicon wafers during semiconductor fabrication. Another application is that the detector may have an important role on a high-resolution mass spectrometer and may help speed up human gene sequencing.

\section{Developmental problems}

The development of a practical "super detector" had a number of major difficulties. One of the first problems was to sort through the various available techniques for converting $\mathrm{x}$-ray radiation into detectable thermal energy. For example, the first $x$-ray microcalorimeters used ultra-low heat capacity semiconducting or superconducting absorbers and semiconductor thermister thermometers. In these devices, absorbed $x$-rays cause a temperature rise in the thermister with the resulting increase in electrical resistance. Unfortunately these devices suffered from an inherently small heat capacity and they were intrinsically slow, with their lower time constant limitation being about $1 \mathrm{~ms}$. A real breakthrough came in 1996 when Irwin et al. developed a superconducting transition-edge sensor (TES) thermometer. The TES employs a superconducting film biased within its normal transition from the normal to the superconducting state. Using this device it 
is possible to build detectors with improved energy resolution using normal-metal absorbers with heat capacities up to two orders of magnitude larger than previously possible. Also the TES detectors have a low impedance, which makes them relatively insensitive to microphonic pickup, thus allowing them to be read out using semiconducting quantum interference device (SQUID) current amplifiers. SQUID detectors are preferred over JFETs in that they are quieter and they dissipate far less power. Also they can be operated at lower temperature, so they can be positioned closer to the detector, thereby minimizing noise pick up. And, most importantly, the TES device lends itself to more simple operation, faster thermal response times and improved dynamic range.

\section{It's got to be cold!}

Although there are many advantages in using the TES detector, there are several problems. The most significant problem is that the detector must be operated at cryogenic temperatures. Where as $\mathrm{Si}(\mathrm{Li})$ and $\mathrm{HPGe}$ EDS detectors are cooled by $\mathrm{LN}_{2}$ to about 100 $K$, it is necessary to cool the NIST TES detectors to the extremely low temperature of about $100 \mathrm{mK}$. To achieve these low temperature, the NIST team designed and constructed a compact and portable adiabatic demagnetization refrigerator (ADR). This refrigerator features a series of thermally isolated stages. The outer stage is a $L N_{2}$ reservoir which cools to $77 \mathrm{~K}$. The middle stage is a LHe reservoir that further cools to $4 \mathrm{~K}$. The ADE innermost layer consists of two or more paramagnetic pills and a superconducting magnet that completes the job of achieving temperatures as low as $50 \mathrm{mK}$. The electronics were designed so that the regulated temperature could be achieved from $55 \mathrm{mK}$ to $4 \mathrm{~K}$.

\section{Other concerns}

The NIST developmenta! team also tackled other significantly problems including concerns about count rates, collection efficiency and other concerns. For example, the count rates in the microcalorimeter are inherently far less than the $50,000 \mathrm{~s}^{-1}$ of the WDS detectors and the $3000 \mathrm{~s}^{-1}$ count limitation of the high resolution semiconductor EDS detectors. However the authors are confident that the count rate can be increased from the initial $150 \mathrm{~s}^{-1}$ to at least $500 \mathrm{~s}^{-1}$ without loss of degradation. Similarly the small size of the microcalorimter detecting elements $\left(0.06 \mathrm{~mm}^{2}\right)$ results in a small geometrical collection efficiency when compared to the WDS and EDS detectors. However with the addition of an $\mathrm{x}$-ray focusing system called the polycapiliary $x$-ray optic, test data showed that the collection efficiency can be increased to where it is larger than typical with the WDS and only a factor of ten smaller than with the high-resolution semiconductor EDS detector.

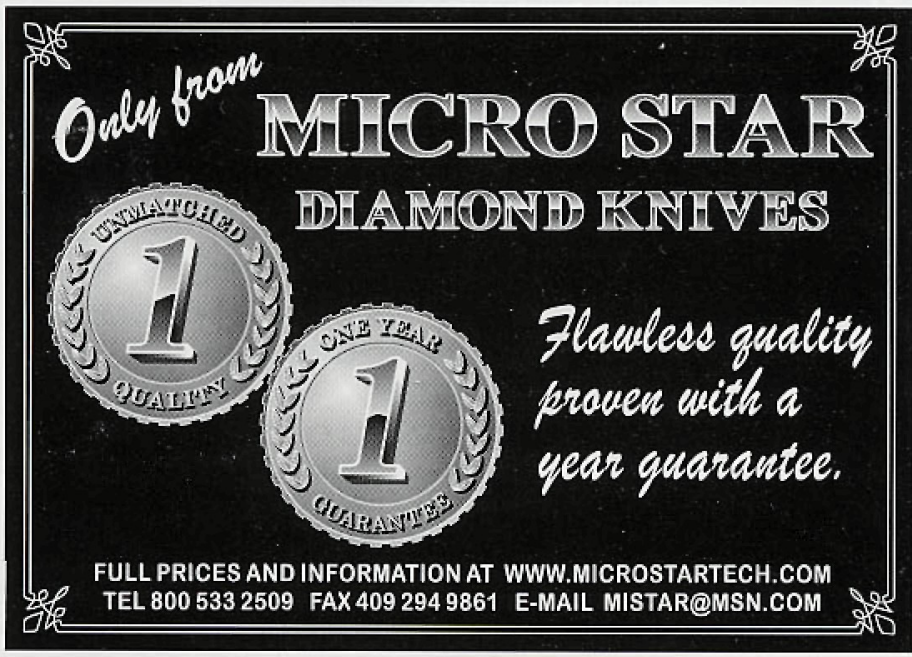

\section{But a commercial product?}

Although most of the technical problems were studied and appropriate solutions proposed by the NIST team, it is a sure bet that there are many other challenges that face the two companies that have received the commercialization licenses. Certainly there will be many production and parts-acquisition problems that will make it difficult to duplicate the NIST development system. The huge size, as well as the involved procedures necessary to operate the system at cryogenic temperatures, may deter some prospective purchasers. To many the system cost, probably exceeding $\$ 250,000$, may be prohibitive. In other words there is a big difference between putting together a system to prove technology and assembling the production parts and know how to produce and market a successful system.

In any respect, it will be an interesting M\&M show, particularly if one of the two licensed companies bites the bullet and introduces the first commercial $\mathrm{x}$-ray microcalorimeter .
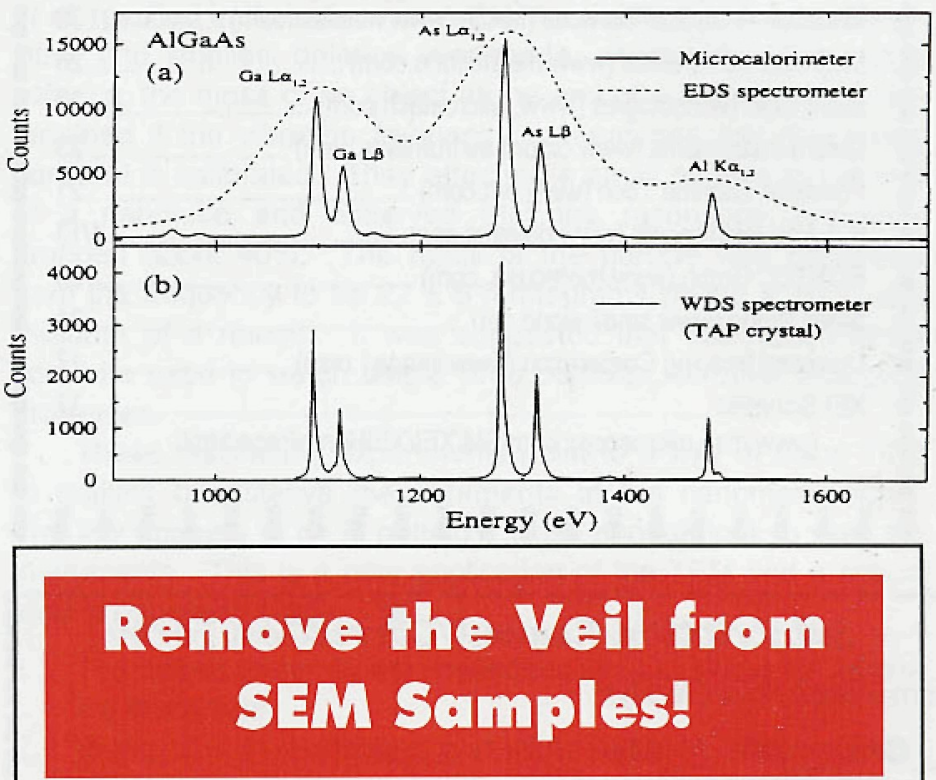

\section{JVACRON B in-sifu Plasma Cleaning stops contamination inside your SEML}

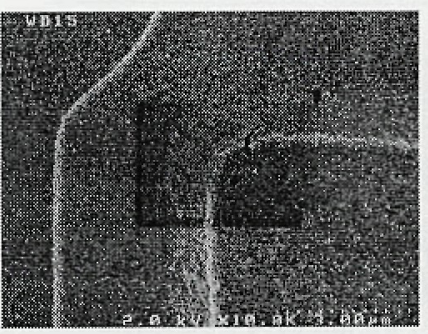

Unwanted Artifact

A short SEM scan started a contamination deposit.

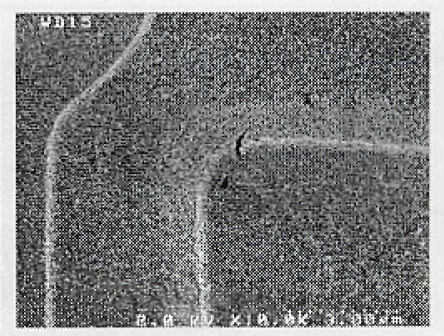

After Evactron Cleaning 2 minutes of cleaning in-situ removed the deposit and prevented new artifacts.

\section{XEI sCIENTIFIC}

SEM-CLEANTM Systems for SEMs

3124 Wessex Way, Redwood City, CA 94061

(650) 369-0133, FAX (650) 363-1659

Email: RVaneXEI@concentric.net Www.EVACTRON.com 